\title{
WEAKLY COMPACT BILINEAR FORMS AND ARENS REGULARITY
}

\author{
A. ÜLGER
}

(Communicated by John B. Conway)

\begin{abstract}
Let $X$ and $Y$ be two Banach spaces. We show that a bounded bilinear form $m: X \times Y \rightarrow \mathbf{C}$ is Arens regular iff it is weakly compact. This result permits us to find very short proofs of some known results as well as some new results. Some of them are: Any $C^{*}$-algebra, the disk algebra and the Hardy class $H^{\infty}$ are Arens regular under every possible product. We also characterize the Arens regularity of certain bilinear mappings.
\end{abstract}

Introduction. The theory of Arens multiplications is usually taken to be about Banach algebras $[4,6,9,21]$. In fact Arens himself presented his theory principally in terms of bilinear mappings [2], though logically the two are equivalent (see $\S 2$ below). Bilinear mappings can be naturally identified with certain linear mappings, and in this paper we show that, by shifting the emphasis to these linear maps, the proofs of most of the main results about the regularity of Arens extensions can be made almost trivial-or perhaps we should say that the real work can be delegated to standard theorems of analysis - and many new results can be obtained. In particular, the theory of weakly compact operators provides new insights, for example the regularity of a Banach algebra is sometimes a property of the geometry of the underiying Banach space rather than of the particular multiplication.

This work is organized as follows. In $\S 1$ we have collected basic definitions, results and notations we will need. In $\S 2$ we prove that a bounded bilinear form defined on the product of two Banach spaces $X$ and $Y$ is Arens regular iff it is weakly compact iff it factors through a reflexive Banach space. This theorem combined with the well-known theorems of analysis, produces, as corollaries, most of the known results about Arens regularity as well as the following results which seem to be new: (i) under every possible product, any $C^{*}$-algebra, the disk algebra $A(D)$ and the Hardy class $H^{\infty}$ are Arens regular. These results extend a result, and answer a question, of [12]. (ii) A bounded bilinear form $m: X \times Y \rightarrow C$ is Arens regular iff its higher Arens extensions $m^{3 n^{*}}$ are Arens regular. In $\S 3$ we characterize the Arens regularity of certain bilinear mappings one encounters very often. In particular, we show that, $A$ being a Banach algebra with a unit element, the bilinear mapping $m:(x, f) \in A \times A^{\prime} \rightarrow{ }_{x} f \in A^{\prime}$ is Arens regular if $A$ is reflexive.

The author would like to express his thanks to Professor J. S. Pym for his encouragement and is grateful to him for valuable conversations.

Received by the editors December 3, 1985 and, in revised form, September 12, 1986.

1980 Mathematics Subject Classification (1985 Revision). Primary 46H05; Secondary 46H99.

Key words and phrases. Arens regularity, weakly compact bilinear forms, $C^{*}$-algebra, disk algebra, Hardy class. 
1. Definitions and notations. Throughout this note, $X, Y, Z$ will be three arbitrary Banach spaces and $A$ an arbitrary Banach algebra. $X^{\prime}$ will denote the dual of $X$ and $\langle x, f\rangle$ the canonical duality between $X$ and $X^{\prime}$. We shall consider $X$ as naturally embedded into $X^{\prime \prime}$. The elements of $X^{\prime}$ will be denoted by $x^{\prime}, y^{\prime}, \ldots$, and sometimes by $f, g, \ldots$; those of $X^{\prime \prime}$ by $x^{\prime \prime}, y^{\prime \prime}, \ldots$ If $K$ is a compact set, the only norm we shall consider on $C(K)$ is the norm of uniform convergence on $K$.

By $B(X, Y)$ we shall denote the space of continuous bilinear forms $m: X \times Y \rightarrow$ $\mathrm{C}$, where $\mathbf{C}$ is the field of complex numbers. Bilinear forms $m \in B(X, Y)$ can be represented as $m(x, y)=\langle u(x), y\rangle=\langle x, v(y)\rangle$ where $u: X \rightarrow Y^{\prime}$ and $v: Y \rightarrow X^{\prime}$ are continuous linear operators with $\|u\|=\|v\|=\|m\|$. This representation provides an isomorphism between the space $B(X, Y)$ and the space $L\left(X, Y^{\prime}\right)$ of continuous linear operators from $X$ into $Y^{\prime}$.

A continuous linear operator $u: X \rightarrow Z$ is said to be weakly compact if the image of the unit ball of $X$ under $u$ is relatively compact for the weak topology $\sigma\left(Z, Z^{\prime}\right)$ of $Z$. Now let $m \in B(X, Y)$ be a bilinear form and $u \in L\left(X, Y^{\prime}\right)$ (resp. $v \in L\left(Y, X^{\prime}\right)$ ) be the linear operator representing $m$. One can see very easily that $u$ is weakly compact iff $v$ is. We shall use the definition:

DEFINITION 1.1. A bilinear form $m \in B(X, Y)$ is said to be "weakly compact" if the linear operator $u \in L\left(X, Y^{\prime}\right)$ representing $m$ is weakly compact.

Now, let again $u: X \rightarrow Y^{\prime}$ be a continuous linear operator. $u$ is said to be "reflexive" (hilbertian) if there exist a reflexive (Hilbert) space $R$ and two continuous linear operators $\phi: X \rightarrow R$ and $\theta: R \rightarrow Y^{\prime}$, such that $\theta \circ \phi=u$. Then the adjoint $\theta^{*}$ of $\theta$ applies $Y^{\prime \prime}$ into $R^{\prime}$; in particular $\theta^{*}(Y) \subseteq R^{\prime}$. This motivates the next definition.

DEFinition 1.2. A bilinear form $m \in B(X, Y)$ is said to be "reflexive" (hilbertian) if there exist a reflexive (Hilbert) space $R$ and two continuous bilinear operators $\phi: X \rightarrow R$ and $\psi: Y \rightarrow R^{\prime}$ such that $m(x, y)=\langle\phi(x), \psi(y)\rangle$.

If $m: X \times Y \rightarrow Z$ is a bounded bilinear mapping the Arens extensions of $m$ will be denoted, as in [2], by $m^{* * *}$ and $m^{t * * * t} . m$ is said to be "Arens regular" or simply "regular" if $m^{* * *}=m^{t * * * t}$. Arens has shown [2] that $m$ is regular iff, for each $h \in Z^{\prime}$, the bilinear form $h \circ m$ is regular. This result reduces the regularity problem of bilinear mappings to that of bilinear forms. We shall use this result without further reference.

Finally, we give a few notations and terminologies about Banach algebras. A Banach algebra $A$ is said to be "Arens regular" if its product $m(x, y)=x y$ considered as a bilinear mapping $m: A \times A \rightarrow A$ is regular. If $x \in A$ and $f \in A^{\prime}$, ${ }_{x} f: A \rightarrow \mathbf{C}$ is the linear operator defined by ${ }_{x} f(y)=f(x y)$. The functional $f$ is said to be "weakly almost periodic" (wap) if the mapping $x \in A \rightarrow{ }_{x} f \in A^{\prime}$ is weakly compact.

The interested reader may find ample information and references about the Arens regularity problem in the survey paper [9].

2. Weakly compact bilinear forms and Arens regularity. In this section we first give an alternative construction of the Arens extensions $m^{* * *}$ and $m^{t * * * t}$ of a bilinear form $m \in B(X, Y)$. To this end, let $u: X \rightarrow Y^{\prime}\left(v: Y \rightarrow X^{\prime}\right)$ be the linear operator representing $m . u^{*}$ and $u^{* *}$ will denote the first and second adjoints of $u$, respectively. Now we define two bounded bilinear forms on $X^{\prime \prime} \times Y^{\prime \prime}$ by

$$
\Phi_{m}\left(x^{\prime \prime}, y^{\prime \prime}\right)=\left\langle u^{* *}\left(x^{\prime \prime}\right), y^{\prime \prime}\right\rangle \quad \text { and } \quad \Psi_{m}\left(x^{\prime \prime}, y^{\prime \prime}\right)=\left\langle x^{\prime \prime}, v^{* *}\left(y^{\prime \prime}\right)\right\rangle \text {. }
$$


We have the following result:

Proposition 2.1. Let $m \in B(X, Y)$. Then $m^{* * *}=\Phi_{m}$ and $m^{t * * * t}=\Psi_{m}$.

PrOOF. We shall prove the first equality only, the proof of the second equality is very similar. To see that this equality holds, we first observe that, when obvious identifications are made, $m$ is identified with $m^{*}: X \rightarrow Y^{\prime}$. Now taking adjoints, we get $m^{* *}: Y^{\prime \prime} \rightarrow X^{\prime}$ and $m^{* * *}: X^{\prime \prime} \rightarrow Y^{\prime \prime \prime}$. The last mapping, interpreted as an element of $B\left(X^{\prime \prime}, Y^{\prime \prime}\right)$, obviously coincides with $\Phi_{m}$.

Next, we give a simple but important characterization of the Arens regularity for bilinear forms.

THEOREM 2.2. Let $m \in B(X, Y)$. Then $T F A E$.

(a) $m$ is Arens regular.

(b) $m$ is weakly compact.

(c) $m$ is reflexive.

Proof. $\quad(\mathrm{a}) \Rightarrow(\mathrm{b})$. Suppose $m$ is regular and let $u: X \rightarrow Y^{\prime}$ be the linear operator representing $m$. By Theorem 3.3 of [2], $\Phi_{m}$ is separately $w^{*}$-continuous on $X^{\prime \prime} \times Y^{\prime \prime}$. Since

$$
\Phi_{m}\left(x^{\prime \prime}, y^{\prime \prime}\right)=\left\langle u^{* *}\left(x^{\prime \prime}\right), y^{\prime \prime}\right\rangle=\left\langle x^{\prime \prime}, u^{*}\left(y^{\prime \prime}\right)\right\rangle
$$

we conclude that $u^{*}: Y^{\prime \prime} \rightarrow X^{\prime}$ is continuous for the topologies $\sigma\left(Y^{\prime \prime}, Y^{\prime}\right)$ and $\sigma\left(X^{\prime}, X^{\prime \prime}\right)$. This implies [10, VI.4.7] that $u$ is weakly compact.

(b) $\Rightarrow$ (c). Suppose $m$ is weakly compact, and let $u: X \rightarrow Y^{\prime}$ be the linear operator representing $m$. Then, by the Davis-Figiel-Johnson-Pełczyński factorization theorem [7], there exist a reflexive space $R$ and two continuous linear operators $\phi: X \rightarrow R$ and $\theta: R \rightarrow Y^{\prime}$ such that $\theta \circ \phi=u$. Let $\psi$ be the restriction of $\theta^{*}$ to $Y$. Then $m(x, y)=\langle\theta \circ \phi(x), y\rangle=\langle\phi(x), \psi(y)\rangle$ and $m$ is reflexive (Definition 1.2 above).

(c) $\Rightarrow(\mathrm{a})$. Suppose $m$ is reflexive. Then, by definition, there exist a reflexive space $R$ and two continuous linear operators $\phi: X \rightarrow R$ and $\psi: Y \rightarrow R^{\prime}$ such that $m(x, y)=\langle\phi(x), \psi(y)\rangle$. Obviously, $\Phi_{m}\left(x^{\prime \prime}, y^{\prime \prime}\right)=\left\langle\phi^{* *}\left(x^{\prime \prime}\right), \psi^{* *}\left(y^{\prime \prime}\right)\right\rangle$. The reflexivity of $R$ implies that $\Phi_{m}$ is separately $w^{*}$-continuous on $X^{\prime \prime}$ and $Y^{\prime \prime}$. Now, by Theorem 3.3 of [2] we conclude that $m$ is regular.

Theorem 2.2 has several corollaries. We proceed with some of them. The first result is about the regularity of the Arens extension of a regular bilinear form. According to the Gantmacher theorem [10, VI.4.8] a continuous linear operator $u: X \rightarrow Y^{\prime}$ is weakly compact iff its adjoint $u^{*}$ is weakly compact. This result, combined with Proposition 2.1 and Theorem 2.2, gives the corollary:

COROLlaRY 2.3. A bilinear form $m \in B(X, Y)$ is Arens regular iff $m^{* * *}$ is Arens regular iff $m^{3 n^{*}}(n=2,3, \ldots)$ is Arens regular.

This result contrasts with the situation for Banach algebras where the second dual of an Arens regular algebra need not be Arens regular [22, p. 108 or 18, p. 32]. We remark that the corollary remains valid for a bounded bilinear mapping $m: X \times Y \rightarrow Z$ if $Z$ is reflexive.

Theorem 2.2 also yields results on quotients and subspaces. 
COROLlaRY 2.4. Let $X_{0}, Y_{0}$ be closed subspaces of $X$ and $Y$, respectively.

(a) If $m \in B(X, Y)$ is Arens regular, then so is its restriction to $X_{0} \times Y_{0}$.

(b) Let $m_{0} \in B\left(X / X_{0}, Y / Y_{0}\right)$ and let $m$ be the composition of $m_{0}$ with the canonical mapping $X \times Y \rightarrow\left(X / X_{0}\right) \times\left(Y / Y_{0}\right)$. Then $m$ is Arens regular iff $m_{0}$ is Arens regular.

ProOF. (a) is trivial. To prove (b), let $\phi: X \rightarrow X / X_{0}$ (resp. $\psi: X \rightarrow Y / Y_{0}$ ) be the quotient mapping. Then $\phi$ maps the unit ball of $X$ onto that of $X / X_{0}$, and the adjoint $\psi^{*}$ of $\psi$ is an isometry from $\left(Y / Y_{0}\right)^{\prime}$ onto the annihilator $Y_{0}^{\perp}$ of $Y_{0}$ into $Y^{\prime \prime}$. Now let $u: X \rightarrow Y^{\prime}$ (resp. $u_{0}: X / X_{0} \rightarrow\left(Y / Y_{0}\right)^{\prime}$ ) be the linear operator corresponding to $m$ (resp. $m_{0}$ ). From the equalities

$$
m(x, y)=\langle u(x), y\rangle=\left\langle u_{0} \circ \phi(x), \psi(x)\right\rangle=m_{0}(\phi(x), \psi(y))
$$

we deduce that $u=\psi^{*} \circ u_{0} \circ \phi$. The above-mentioned properties of $\phi$ and $\psi$ imply that $u$ is weakly compact iff $u_{0}$ is weakly compact. Now an appeal to Theorem 2.2 completes the proof.

This result, applied to Banach algebras, shows that if $A$ is Arens regular, then any subalgebra and any quotient algebra of $A$ are Arens regular (cf. [6]). In the reverse direction, the Arens regularity of each separable subalgebra of $A$ implies that of $A$. On the other hand, the existence of regular algebras with irregular second duals shows that a second dual algebra may be irregular even if it has a $w^{*}$-dense regular subalgebra.

Standard double limit criteria for weak compactness [14, Theorem 17.12] now yield immediately the "Double Limit Criterion" for regularity.

COROLLARY 2.5. $m \in B(X, Y)$ is regular iff for any two bounded sequences $\left(x_{p}\right)$ and $\left(y_{p}\right)$ in $X$ and $Y$, respectively, the iterated limits $\lim _{p} \lim _{q} m\left(x_{p}, y_{q}\right)$ and $\lim _{p} \lim _{q} m\left(x_{p}, y_{q}\right)$ are equal provided that they both exist.

Next we return to the regularity problem for Banach algebras. Before proceeding, we remark that, as it has been said in the introduction, the regularity problem for bilinear mappings and algebras are in fact equivalent. Indeed, if $m: X \times Y \rightarrow Z$ is a bounded bilinear mapping, we can make $B=X \oplus Y \oplus Z$ into a Banach algebra defining the norm by $\|(x, y, z)\|=\|x\|+\|y\|+\|z\|$ and the product by $\left(x_{1}, y_{1}, z_{1}\right) \cdot\left(x_{2}, y_{2}, z_{2}\right)=\left(0,0, m\left(x_{1}, y_{2}\right)\right)$. One can show very easily that $m$ is regular iff $B$ is regular.

Now, let $m(x, y)=x y$ be the product of the Banach algebra $A$. Then, for $f \in A^{\prime}, f \circ m(x, y)=f(x y)=\left\langle{ }_{x} f, y\right\rangle$. This equality shows that the linear operator $u: A \rightarrow A^{\prime}$ representing $f \circ m$ is given by $u(x)={ }_{x} f$. It follows that $u$ is weakly compact iff $f$ is wap. In view of Corollary 2.5 , the next result--Theorem of $[19]$ - is now merely a tautology.

COROLlary 2.6. A is Arens regular iff each $f \in A^{\prime}$ is wap iff for each $f \in$ $A^{\prime}$, and for any two bounded sequences $\left(x_{p}\right)$ and $\left(y_{p}\right)$ in $A$, the iterated limits, $\lim _{p} \lim _{q} f\left(x_{p} y_{q}\right)$ and $\lim _{q} \lim _{p} f\left(x_{p} y_{q}\right)$ are equal provided that they both exist.

The Arens regularity of an algebra being, by definition, that of the bilinear mapping $m(x, y)=x y$, neither the associativity of the product nor the inequality $\|x y\| \leq\|x\|\|y\|$ plays any role in the regularity of the algebra. For this reason, the following definition seems to be more general and more appropriate for our purpose. 
DEFINITION 2.7. By a "product" on a Banach space $X$ we shall mean a continuous bilinear mapping $p: X \times X \rightarrow X$. Then, the couple $(X, p)$ is said to be "Arens regular" iff $p$ is Arens regular.

H. Haagerup [13] extending a result of G. Pisier [17] has recently shown that if $A, B$ are two $C^{*}$-algebras then any bounded bilinear form $m: A \times B \rightarrow \mathrm{C}$ is hilbertian (Definition 1.2 above). This result, combined with Theorem 2.2, yields the next result.

THEOREM 2.8. Let $A$ be a $C^{*}$-algebra. Then, for every product $p,(A, p)$ is Arens regular.

This theorem generalizes a result of Gulick [12], who has shown that for any product for which $C(K)$ - the space of continuous functions on a compact $K$, endowed with the supremum norm - is a commutative Banach algebra, is regular. In the same paper (p. 136, Question 1) Gulick asks the question: Is the disk algebra $A(D)$-the algebra of continuous functions on the closed unit disk $D$ of $\mathbf{C}$ which are analytic in the interior of $D$, endowed with the supremum norm-Arens regular under any commutative multiplication which makes $A(D)$ a Banach algebra? Actually more is true.

THEOREM 2.9. For every product $p,(A(D), p)$ is Arens regular.

Proof. Let $T$ be the unit circle, i.e. the boundary of $D$. J. Bourgain $[5$, Theorem 1] has shown that any bounded bilinear form $m: A(D) \times A(D) \rightarrow \mathrm{C}$ can be extended to a bounded bilinear form $\tilde{m}: C(T) \times C(T) \rightarrow \mathrm{C}$. By Theorem 2.9 above $\tilde{m}$ is regular. Now Corollary 2.4(a) above shows that $m$ is regular. It follows that any bounded bilinear mapping $p: A(D) \times A(D) \rightarrow A(D)$ is regular.

Another result due to J. Bourgain [16, p. 252] shows that any bounded bilinear form $m: H^{\infty} \times H^{\infty} \rightarrow \mathbf{C}$, where $H^{\infty}$ is the Hardy class, is hilbertian. This result, combined with Theorem 2.2 , yields the next result.

THEOREM 2.10. For every product $p,\left(H^{\infty}, p\right)$ is Arens regular.

The preceding theorems show that the Arens regularity of a $C^{*}$-algebra, $A(D)$ or $H^{\infty}$ does not depend on the particular product of these spaces; but it is rather a property of the geometry of the underlying Banach spaces.

Parallel to the preceding results, there exist distinct Banach spaces $X, Y$ and $Z$ such that any bounded bilinear form $m: X \times Y \rightarrow Z$ is Arens regular. Before stating one or two results in this direction we recall two definitions: A Banach space $X$ is said to be a "Grothendieck space" if each $w^{*}$-convergent sequence $\left(x_{n}^{\prime}\right)$ in $X^{\prime}$ is $\sigma\left(X^{\prime}, X^{\prime \prime}\right)$-convergent. We recall that $l^{\infty}=C(\beta \mathrm{N})$ is a Grothendieck space. More generally, if $K$ is a quasi-stonian compact space (i.e. the closure of any open $F_{\sigma}$-set is open) $C(K)$ is a Grothendieck space, $[\mathbf{2 0}$, p. 131] (see also [11, Theorem 9]). The second definition we recall is that of WCG (weakly compactly generated) Banach spaces. A Banach space $X$ is said to be WCG if it contains a weakly compact set $K$ such that span $K$ is dense in $X$. We recall that any separable Banach space, the space $c_{0}(\Gamma)$ for any set $\Gamma$ are WCG. Now we can state the following result.

THEOREM 2.11. Let $X$ be a Grothendieck space, $Y$ a Banach space whose dual is WCG, and $Z$ an arbitrary Banach space. Then any bounded bilinear mapping $m: X \times Y \rightarrow Z$ is Arens regular. 
ProOF. Let $m: X \times Y \rightarrow Z$ be a bounded bilinear mapping and $h \in Z^{\prime}$. Denote by $u: X \rightarrow Y^{\prime}$ the linear operator representing $h \circ m$. $X$ being a Grothendieck space and $Y^{\prime}$ WCC, $u$ is weakly compact $[8$, p. 150]. It follows that $m$ is Arens regular.

For example, any bounded bilinear form $m: l^{\infty} \times c_{0} \rightarrow Z$ is Arens regular. But this result can also be deduced from Theorem 2.8 above. Another interesting case of automatic regularity is the following.

THEOREM 2.12. Let $A$ be a $C^{*}$-algebra, and assume that $Y^{\prime}$ is $\sigma\left(Y^{\prime}, Y^{\prime \prime}\right)$ sequentially complete. Then, for any Banach space $Z$, every bounded bilinear mapping $m: A \times Y \rightarrow Z$ is Arens regular.

ProOF. Let $m: A \times Y \rightarrow Z$ be a bounded bilinear mapping and $h \in Z^{\prime}$. Then the linear operator $u: A \rightarrow Y^{\prime}$ representing $h \circ m$ is weakly compact, [1, Theorem 4.2]. Hence, by Theorem 2.2 above, we conclude that $m$ is regular.

If, in this theorem, we take $A=Y=C(K)$ then $Y^{\prime}$, being isomorphic to an $L$ space $[\mathbf{1 4}, \mathbf{2 0}]$, is weakly sequentially complete. It follows that any bounded bilinear mapping $m: C(K) \times C(K) \rightarrow Z$ is Arens regular. This is, of course, a particular case of Theorem 2.8 above. But, independently from Theorem 2.8, Theorem 2.12 shows that, under every product, $C(K)$ is Arens regular. For instance, if $G$ is a compact group, $C(G)$ with the convolution as the multiplication is Arens regular. The same conclusion applies to $L^{\infty}(G)$.

3. Arens regularity of certain bilinear mappings. In this section we characterize the Arens regularity of certain bilinear mappings one encounters very often. The main result of this section is the following.

THEOREM 3.1. Let $A$ be a Banach algebra with a unit element. Then, the bilinear mapping $m:(x, f) \in A \times A^{\prime} \rightarrow{ }_{x} f \in A^{\prime}$ is Arens regular iff $A$ is reflexive.

Proof. Assume $m$ is regular and let $e$ be the unit element of $A$. Define $\hat{e}: A^{\prime} \rightarrow$ $\mathrm{C}$ by $\hat{e}(f)=f(e)$. Then the bilinear form $\hat{e} \circ m$, given by $\hat{e} \circ m(x, f)=f(x)=\langle f, x\rangle$, is weakly compact. This means that the linear operator $v: A^{\prime} \rightarrow A^{\prime}$ representing $\hat{e} \circ m$, which is nothing but the identity operator of $A^{\prime}$, is weakly compact. It follows that $A^{\prime}$, so $A$, is reflexive. The converse is trivial.

In the course of the preceding proof we have incidentally obtained the following simple but very useful result.

COROLLARY 3.2. Let $X$ be an arbitrary Banach space. Then the bilinear form $m:(x, f) \in X \times X^{\prime} \rightarrow f(x) \in \mathbf{C}$ is Arens regular iff $X$ is reflexive.

As an application of the preceding results, let us consider the Arens regularity of the module product: $(a, b) \in A \times l^{1}(A) \rightarrow a b \in l^{1}(A)$, where $l^{1}(A)=\{b=$ $\left.\left(b_{n}\right): b_{n} \in A,\|b\|=\sum_{n=0}^{\infty}\left\|b_{n}\right\|<\infty\right\}$ and $a b=\left(a b_{n}\right)$. The space $l^{1}(A)$, endowed with the given $l^{1}$-norm, is a Banach space and its dual is $l^{\infty}\left(A^{\prime}\right)[\mathbf{1 5}$, p. 359], the space of bounded sequences in $A^{\prime}$. Now, we can give a short proof of the following result, which is Theorem 4 of [3].

COROLlaRY 3.3. Let $A$ be a Banach algebra with a unit element. Then the module product $m:(a, b) \in A \times l^{1}(A) \rightarrow a b \in l^{1}(A)$ is Arens regular iff $A$ is reflexive.

Proof. Assume $A$ is not reflexive. Then, by Corollaries 3.2 and 2.5 above there exist bounded sequences $\left(a_{p}\right)$ in $A$ and $\left(f_{q}\right)$ in $A^{\prime}$ such that the iterated 
limits $\lim _{p} \lim _{q} f_{q}\left(a_{p}\right)$ and $\lim _{q} \lim _{p} f_{q}\left(a_{p}\right)$ exist and are distinct. Now, define the sequence $\left(e_{q}\right)$ in $l^{1}(A)$ by $e_{q}=(0,0, \ldots, e, 0, \ldots)$ ( $e$ is in $q$ th place). Then, $g=\left(f_{n}\right)$ is in the dual space of $l^{1}(A)$ and $g \circ m\left(a_{p}, e_{q}\right)=f_{q}\left(a_{p}\right)$. This equality shows that $g \circ m$, so $m$, is not Arens regular. The converse is trivial.

We do not know whether Theorem 3.1 is valid without the assumption that $A$ has a unit element. However, if $A$ has an approximate identity then the same conclusion holds. Indeed, consider $A^{\prime \prime}$ as a Banach algebra with the first Arens product, i.e. the product which is $w^{*}$-continuous in the left-hand variable. Then $A^{\prime \prime}$ has a right identity $E\left[6\right.$, Lemma 3.8]. Now, $E \circ m(x, f)=E\left({ }_{x} f\right)=f(x)$ (see the proof of Lemma 3.8 of [6]). Then, an application of Corollary 3.2 above shows that $m$ is regular iff $A$ is reflexive. Now, we can prove the next result very easily.

COROLlaRY 3.4. Let $G$ be a locally compact unimodular group endowed with a right invariant Haar measure. Consider the group algebra $A=L^{1}(G)$. Then the convolution operator $m:(x, f) \in L^{1}(G) \times L^{\infty}(G) \rightarrow x * f \in L^{\infty}(G)$ is Arens regular iff $G$ is finite.

ProOF. Let $x \in L^{1}(G)$ and $f \in L^{\infty}(G)$. Since $G$ is unimodular, the function $\tilde{x}$, defined by $\tilde{x}(t)=x\left(t^{-1}\right)$, is in $L^{1}(G)$. An easy calculation shows (see [6, p. 856]) that $\tilde{x} f=x * f$. Since the group algebra $L^{1}(G)$ has an approximate identity, we conclude from the preceding remark that the bilinear mapping $m$ is Arens regular iff $A$ is reflexive iff $G$ is finite.

We remark that, unless $G$ is finite, the group algebra $L^{1}(G)$ is not Arens regular $[6,23]$.

In $\S 2$ we have seen that there exist Banach spaces $X, Y$ with the property that any bounded bilinear form $m: X \times Y \rightarrow \mathbf{C}$ is Arens regular. The next result, which is our last result, shows that this property is equivalent to the regularity of a single bilinear mapping $\phi: X \times Y \rightarrow X \hat{\otimes} Y$.

Proposition 3.5. Let $X, Y$ be two arbitrary Banach spaces and $X \hat{\otimes} Y$ their projective tensor product. Then the canonical bilinear mapping $\phi: X \times Y \rightarrow X \hat{\otimes} Y$ (i.e. $(x, y)=x \otimes y)$ is Arens regular iff each bounded bilinear form $m: X \times Y \rightarrow \mathbf{C}$ is Arens regular.

PROOF. The projective tensor product is defined in such a way that the dual space of $X \hat{\otimes} Y$ and $B(X, Y)$ are canonically isomorphic. Under the canoncial isomorphism, to an $f$ in $(X \hat{\otimes} Y)^{\prime}$ there corresponds the bilinear form $m=f \circ \phi$. From this the conclusion follows.

The author is grateful to the referee for his helpful remarks.

\section{REFERENCES}

1. C. A. Akemann, P. G. Dodds and J. L. B. Gamlen, Weak compactness in the dual space of $C^{*}$-algebra, J. Funct. Anal. 10 (1972), 446-450.

2. R. Arens, The adjoint of a bilinear operation, Proc. Amer. Math. Soc. 2 (1951), 839-848.

3. N. Arikan, Arens regularity and reflexivity, Quart. J. Math. Oxford Ser. 32 (1981), 383-388.

4. F. F. Bonsall and J. Duncan, Complete normed algebras, Springer-Verlag, Berlin and New York, 1973.

5. J. Bourgain, New Banach space properties of certain spaces of analytic functions, Proc. Internat. Congress of Mathematicians, vol. II, Warzawa, 1983, pp. 945-951.

6. P. Civin and B. Yood, The second conjugate space of a Banach algebra as an algebra, Pacific J. Math. 11 (1961), 847-870. 
7. W. J. Davis, T. Figiel, W. B. Johnson and A. Pełczyński, Factoring weakly compact operators, J. Funct. Anal. 17 (1974), 311-327.

8. J. Diestel, Geometry of Banach spaces-Selected topics, Lecture Notes in Math., vol. 485, Springer-Verlag, Berlin and New York, 1975.

9. J. Duncan and S. A. R. Hosseinium, The second dual of a Banach algebra, Proc. Edinburgh Math. Soc. 84A (1979), 309-325.

10. N. Dunford and J. T. Schwartz, Linear operators, Part I, Interscience, New York, 1958.

11. A. Grothendieck, Sur les applications linéaires faiblement compactes d'espaces du type $C(K)$, Canad. J. Math. 5 (1953), 129-173.

12. S. L. Gulick, Commutativity and ideals in the biduals of topological algebras, Pacific J. Math. 18 (1966), 121-137.

13. U. Haagerup, The Grothendieck inequality for bilinear forms on $C^{*}$-algebras, Adv. in Math. 56 (1985), 93-116.

14. J. L. Kelly and I. Namioka, Linear topological spaces, Van Nostrand, Princeton, N.J., 1963.

15. G. Köthe, Topological vector spaces. I, Springer-Verlag, Berlin and New York, 1969.

16. A. Pełczyński, Structural theory of Banach spaces and its interplay with analysis and probability, Proc. Internat. Congress of Mathematicians, vol. I, Warzawa, 1983, pp. 237-269.

17. J. Pisier, Grothendieck's theorem for noncommutative $C^{*}$-algebras with an application on Grothendieck's constants, J. Funct. Anal. 29 (1978), 394-415.

18. J. S. Pym, Remarks on the second duals of Banach algebras, J. Nigerian Math. Soc. 2 (1983), 31-33.

19. The convolution of functionals on spaces of bounded functions, Proc. London Math. Soc. 15 (1965), 84-104.

20. H. H. Schaefer, Banach lattices and positive operators, Springer-Verlag, Berlin and New York, 1974.

21. P. K. Wong, On the Arens products and certain Banach algebras, Trans. Amer. Math. Soc. 180 (1973), 437-448.

22. N. Young, Periodicity of functionals and representations of normed algebras on reflexive spaces, Proc. Edinburgh Math. Soc. 20 (1976), 99-120.

23. N. Young, The irregularity of multiplication in group algebras, Quart. J. Math. Oxford Ser. 24 (1973), 59-62.

Department of Mathematics, BoĞaziçi University, P.K.2, Bebek, Istanbul, TURKEY 